\title{
The Thermal Behavior of Pd on Graphene/Ru(0001)
}

\author{
Cheol-Woo Yi ${ }^{\mathrm{a}, \mathrm{b}}$ and János Szanyi ${ }^{*} \mathrm{~b}$ \\ ${ }^{a}$ Department of Chemistry and Institute of Basic Science, Sungshin Women's University, Seoul \\ 142-732, South Korea \\ ${ }^{b}$ Institute for Integrated Catalysis, Pacific Northwest National Laboratory, P.O. Box 999, MSIN: \\ K8-87, Richland, WA, USA
}

The thermal behavior of various amounts of palladium deposited onto graphene/ $\mathrm{Ru}(0001)$ at room temperature was investigated by x-ray photoelectron spectroscopy (XPS) and infrared reflection absorption spectroscopy (IRAS) of adsorbed CO. IRAS spectra of adsorbed CO on palladium deposited onto the graphene layer reveal the formation of defect-rich, highly-stepped and/or nanoparticle-typed palladium clusters. Annealing to temperatures below $900 \mathrm{~K}$, palladium clusters mainly experience agglomeration on the graphene layer, but at higher temperatures intercalation of palladium between the graphene layer and Ru substrate takes place. Eventually, palladium completely desorbs and the graphene layer dissolves into the ruthenium substrate at $1400 \mathrm{~K}$. Even though the annealing induces the intercalation and desorption of palladium, the topmost graphene layer stays intact without any physical damage at and below $1300 \mathrm{~K}$.

\footnotetext{
* To whom correspondence should be addressed. E-mail: janos.szanyi@pnnl.gov 


\section{Introduction}

Graphene is a single atomic layer consisting of $\mathrm{sp}^{2}$-hybridized carbon. It is expected to be applied in many fields due to its remarkable electronics properties [1]. Because of its importance, the interfaces between graphene and transition metals have been investigated extensively [1,2]. Moreover, the nano-composite materials consisting of graphene and transition metal(s) are attracting a great attention for numerous technological applications in the field of electronics, material science, catalysis and so on [1,2]. The interfaces between graphene and transition metals have been recently investigated [1-5]. Among them, palladium-graphene composite is employed in various chemical sensors and catalytic reactions [6,7]. Investigations on well-defined model systems can greatly contribute to the understanding of the properties of metal overlayers on graphene. The epitaxial growth of high-quality graphene has recently been investigated on various metal and inorganic material surfaces as substrates [2,8]. Despite the great importance of metal particles on graphene substrates, fundamental studies of the physical and chemical properties of palladium clusters on graphene are still needed. Two distinct intercalation mechanisms of metal under the graphene layer were proposed by the strength of interaction between intercalant and carbon [9]. It is believed that the metal, which weakly interact with carbon, such as $\mathrm{Pb}$, is intercalated through extended defects (open edge and/or domain boundaries), but the metal strongly interacted with carbon via exchange mechanism [9]. The intercalation via exchange mechanism needs higher temperature than that through extended defects. In this study, palladium was deposited onto graphene/Ru(0001) surfaces at room temperature, and the thermal behaviors of palladium clusters have been investigated with $\mathrm{x}$-ray photoelectron spectroscopy (XPS), low energy electron diffraction (LEED), and infrared reflection absorption spectroscopy (IRAS) of adsorbed CO. Under low temperature (up to 800 
K) annealing in vacuum, palladium clusters mainly experience agglomeration on the graphene layer. However, further annealing at higher temperature $(\sim 900-1100 \mathrm{~K})$ causes to the intercalation of palladium between the graphene layer and Ru substrate, and then Pd clusters desorb from the substrate at temperatures $>1100 \mathrm{~K}$. Even though the annealing induces the intercalation of palladium between graphene and ruthenium substrate and desorption of palladium, the topmost graphene layer remained intact. This observation is a good agreement with hypothetical exchange mechanism of intercalation.

\section{Experimental}

The experimental setup and the data acquisition procedures have been discussed in detail elsewhere [10-13]. All the experiments were performed in an ultrahigh vacuum (UHV) surface analysis chamber equipped with $\mathrm{x}$-ray photoelectron spectroscopy, low-energy electron diffraction, and quadrupole mass spectrometry, and connected to an elevated-pressure cell equipped with $\mathrm{KBr}$ windows for infrared reflection absorption spectroscopy measurements. The base pressure in both chambers was $\sim 1 \times 10^{-10}$ Torr. The $\mathrm{Ru}(0001)$ single crystal used in these experiments (Princeton Scientific Corp., diameter $=10 \mathrm{~mm}$ and thickness $=1 \mathrm{~mm}$ ) was spotwelded onto a $\mathrm{U}$-shaped Ta wire (0.030 in. diameter). The sample temperature was measured by a C-type thermocouple spot-welded to the backside of the single crystal. The $\mathrm{Ru}(0001)$ single crystal was cleaned by repeated cycles of Ar ion sputtering, oxidation at $1000 \mathrm{~K}$ and annealing up to $1900 \mathrm{~K}$ in UHV, and the cleanliness and order of the surface were verified with XPS and LEED, respectively. The high quality epitaxial graphene layer is prepared by pyrolysis of ethylene on $\mathrm{Ru}(0001)$ surface. The Ru was annealed at $1400 \mathrm{~K}$ for $300 \mathrm{sec}$ and then the sample was rapidly cooled down under ethylene atmosphere $\left(1 \times 10^{-6}\right.$ Torr $)$. The quality of graphene 
layer was monitored with LEED and IRAS with the adsorption of carbon monoxide. Pd was deposited at room temperature onto the graphene layer supported on $\mathrm{Ru}(0001)$ using a resistively heated palladium wire (Alfa Aesar; diameter $=0.25 \mathrm{~mm}$; 99.99\%). The palladium coverage was calibrated with respect to clean Ru by the break point of XPS peak ratio $(\mathrm{Pd} / \mathrm{Ru})$ as shown in Figure $\mathrm{S} 1[14,15]$. Infrared spectra were collected at $4 \mathrm{~cm}^{-1}$ resolution using a grazing angle of approximately $85^{\circ}$ to the surface normal. All the IR spectra collected were referenced to a background spectrum, which is acquired from the sample prior to gas adsorption, and taken under $\mathrm{CO}$ atmosphere $\left(1 \times 10^{-6} \mathrm{Torr}\right)$ at saturation $\mathrm{CO}$ coverage.

\section{Results and discussion}

The quality of the prepared graphene layer was monitored by XPS, LEED, and IRAS. The infrared spectrum obtained from the graphene layer after $\mathrm{CO}$ exposure $\left(1 \times 10^{-6} \mathrm{Torr}\right.$ of $\left.\mathrm{CO}\right)$ at room temperature showed no $\mathrm{CO}$ vibrational features, indicating full and continuous coverage of carbon on the Ru surface (i.e., no exposed Ru atoms were present). Carbon monoxide is one of the best probe molecules to identify defect sites on the surface. Due to the strong interaction of $\mathrm{CO}$ with $\mathrm{Ru}$ even at room temperature, $\mathrm{CO}$ vibrational features should appear once the graphene layer is defective and/or Ru sites are exposed through the "holes" in the graphene. Hence, the sharp LEED pattern (shown in Figure S2 (a) and (b)) and the absence of any CO vibrational feature in the IRAS spectrum unambiguously show that the graphene film is of good

quality and fully covers the ruthenium substrate. After the desired amount of Pd was deposited onto the graphene (GR) layer at room temperature, the sample was annealed step-wise (in $100 \mathrm{~K}$ intervals) and changes in the $\mathrm{Pd} / \mathrm{GR} / \mathrm{Ru}(0001)$ systems were monitored by both XPS and IRAS of adsorbed CO. The samples were kept at each annealing temperature for 5 minutes. Figures 1 
(a) and 2 (a) show the normalized XPS intensities of $\mathrm{Pd}, \mathrm{Ru}$, and $\mathrm{Pd} / \mathrm{Ru}$ in UHV and infrared spectra during $\mathrm{CO}$ exposure $\left(1.0 \times 10^{-6}\right.$ Torr of $\left.\mathrm{CO}\right)$ for 0.25 monolayer equivalent (MLE) $\mathrm{Pd} / \mathrm{GR} / \mathrm{Ru}(0001)$ system as a function of annealing temperature, respectively. In the temperature range of $300-900 \mathrm{~K}$, the XPS intensity of Pd gradually decreases with increasing annealing temperature. This phenomenon is attributed to the agglomeration of Pd clusters deposited onto the graphene layer as the sample temperature was increased. The surface free energy of Pd is reported to be $2.043 \mathrm{~J} / \mathrm{m}^{2}$ [15] which is significantly higher than that of graphene, $4.67 \times 10^{-2}$ $\mathrm{J} / \mathrm{m}^{2}$ [16]. The deposited Pd forms three-dimensional clusters on the graphene layer due to the difference of surface free energies and the sufficient mobility of $\mathrm{Pd}$ atoms [17]. Moreover, N'Diaye et al. [18,19] and Batzill [1] reported that the formation and stability of metal cluster on graphene moiré structure depend on several parameters such as lattice constant and cohesive energies of the deposited materials as well as the interaction between the metal and the graphene. N'Diaye et al. observed the high stability of $\operatorname{Ir} /$ graphene/Ir(111) and suggested the extra stabilization owing to the formation of strong metal-carbon bond via $\mathrm{sp}^{2} \rightarrow \mathrm{sp}^{3}$ re-hybridization of carbon below the metal cluster $[1,18,19]$. Hence, the dissociation energy $(436 \mathrm{~kJ} / \mathrm{mol})$ of $\mathrm{Pd}-$ $\mathrm{C}$ bond is relatively lower than that of other transition metals, such as $\operatorname{Ir}(631 \mathrm{~kJ} / \mathrm{mol}), \mathrm{Pt}(610$ $\mathrm{kJ} / \mathrm{mol})$, and $\mathrm{Rh}(580 \mathrm{~kJ} / \mathrm{mol})$ which form highly dispersed clusters on graphene layers [17]. As a result, the normalized XPS signal of ruthenium maintains an almost constant value, even though that of Pd gradually decreases. This observation is consistent with the IRAS results of adsorbed $\mathrm{CO}$ shown in Figure 2 (a). The agglomeration of Pd clusters reduces the number of adsorption sites of $\mathrm{CO}$ due to the reduction in surface area. Hence, the infrared intensity of $\mathrm{CO}$ gradually decreases without change in the pattern of IRAS spectrum. The IR intensity of adsorbed $\mathrm{CO}$ on the surface annealed at $900 \mathrm{~K}$ is reduced by $\sim 25 \%$ in comparison to that on the 
as-deposited surface. It is noteworthy that the vibrational feature of bridge-bound $\mathrm{CO}$ appears at $\sim 1975 \mathrm{~cm}^{-1}$ which is substantially higher than that of CO bridge-bound on $\operatorname{Pd}(111)$ terrace sites $\left(\sim 1950 \mathrm{~cm}^{-1}\right)$. This is characteristic of bridge-bound CO on defect-rich and/or highly-stepped $\operatorname{Pd}(111)$ facets [20]. In addition, the surfaces of alumina-supported palladium clusters (mean particle size $\sim 3.5 \mathrm{~nm}$ ) grown at $90 \mathrm{~K}$ have been shown to be very defective with no distinct facets, resulting in a blue-shifted vibrational feature of bridge-bound CO [20,21]. Hence, the CO vibrational feature appeared at $1975 \mathrm{~cm}^{-1}$ is a strong evidence for the formation of small palladium clusters on graphene at low Pd loadings. Moreover, it is interesting that the overall shape of the IR spectra did not change much, even though the annealing temperature of the sample was increased from 300 to $900 \mathrm{~K}$. The only difference observed was the decrease in the intensity of the $\mathrm{CO}$ vibrational feature. It indicates that the Pd cluster size on the graphene substrate slightly increases with increasing annealing temperature due to the weak interaction between palladium clusters and the graphene layer. Gotterbarm et. al [22] also observed the agglomeration of $\mathrm{Pd}$ on graphene/Rh(111) during annealing, where the interaction between graphene and substrate is weaker than that of graphene $/ \mathrm{Ru}(0001)$. They proposed that $\mathrm{Pd}$ clusters are agglomerated on the graphene layer as the temperature was raised to $750 \mathrm{~K}$ since the XPS intensity of Pd gradually decreased and exhibited a minimum value at $750 \mathrm{~K}$ [17]. Further annealing caused the intercalation of Pd between the graphene layer and the Rh substrate [17]. In our study, the XPS intensity of Pd and the Pd/Ru intensity ratio have both displayed minimum values at an annealing temperature of $900 \mathrm{~K}$, which is higher than that observed for the $\mathrm{Pd} / \mathrm{GR} / \mathrm{Rh}(111)$ system (750 K) [13]. This difference can be attributed to the stronger interaction between the graphene layer and the $\mathrm{Ru}(0001)$ substrate in our system, which results in higher energy needed for Pd to intercalate between the graphene layer and the Ru metal substrate. 
Above $900 \mathrm{~K}$ annealing temperature, however, the XPS intensity of Pd abruptly increases up to $1100 \mathrm{~K}$ and that of Ru concomitantly shows a gradual decrease. The IRAS spectrum of adsorbed $\mathrm{CO}$ shows a significant reduction in intensity after annealing at $1000 \mathrm{~K}$. Eventually, the CO vibrational feature completely disappears as the sample is annealed to $1100 \mathrm{~K}$. It is indicated that there is no Pd on graphene layer and the graphene layer does not have any defect sites at all. Even though the CO feature is completely disappeared in the IRAS, the XPS intensity of Pd restores almost to the same value as its original intensity was, as shown in Figure 2 (a). These observations are the direct evidences that the Pd deposited onto the graphene layer is intercalated between the graphene layer and the Ru substrate without any damage of graphene layer. The gradual decrease in XPS signal of $\mathrm{Ru}$ is demonstrated that spreading of the intercalated palladium layer occurred on the Ru substrate under the graphene layer. As mentioned above, the interaction between graphene and ruthenium is stronger than that of graphene and rhodium $[3,4,17]$, and therefore, the temperature at which intercalation of palladium occurs is higher in our system, than in the case of $\mathrm{GR} / \mathrm{Rh}(111)$. Further annealing the sample above $1100 \mathrm{~K}$ causes the reduction of Pd and increase in Ru XPS intensities due to the onset of Pd desorption at this temperature, as it has been shown for Pd overlayers on refractory metals $[23,24]$. The onset of multilayer Pd desorption is $\sim 1100 \mathrm{~K}$, while $\mathrm{Pd}$ monolayer desorbs at $\sim 1300 \mathrm{~K}[23,24]$. Therefore, the increase in the intensity of Ru XPS signal above $1100 \mathrm{~K}$ is attributed to the desorption of palladium. Despite desorption of a portion of the palladium intercalated between the graphene layer and the $\mathrm{Ru}$ substrate, the IRAS spectrum after $\mathrm{CO}$ exposure shows no vibrational features. These results unambiguously show that the graphene layer remained intact on the Ru substrate without any measurable damage during the processes of intercalation and desorption of Pd. Annealing at $1400 \mathrm{~K}$ causes the complete desorption of palladium from the Ru 
substrate and the dissolution of the graphene layer into Ru bulk. The Pd XPS feature completely disappears after annealing at $1400 \mathrm{~K}$ and the $\mathrm{CO}$ vibrational feature in the IRAS spectrum appears with high intensity at $2055 \mathrm{~cm}^{-1}$, assigned to adsorbed $\mathrm{CO}$ on $\mathrm{Ru}(0001)[25,26]$.

The thermal behavior of Pd clusters on graphene were further investigated as a function of Pd coverage. Panels (b) - (d) of Figure 1 show the normalized XPS intensities of Pd and Ru, as well as the $\mathrm{Pd} / \mathrm{Ru}$ intensity ratio for various coverages of $\mathrm{Pd}(1.0-5.0 \mathrm{MLE})$ on graphene as a function of annealing temperature, respectively. Despite different Pd coverages on graphene, the variation of XPS intensities of Pd shows a similar trend: it gradually decreases with increasing annealing temperature in the lower temperature region $(300-900 \mathrm{~K})$. However, further annealing, but still below the desorption temperature of palladium, causes an increase in Pd XPS intensity. Eventually, at high annealing temperatures (above $1100 \mathrm{~K}$ ) a steep decrease in the XPS intensity of Pd is observed due to the onset of palladium desorption. At low Pd coverages ( $\leq 1.0 \mathrm{MLE}$ ), the thermal behavior of the metal overlayers as a function of annealing temperature is very similar. However, as the Pd coverage increases above 1 MLE, the temperature having minimum Pd XPS intensity decreases and the variation of Pd intensity is more pronounced due to the facile agglomeration of large Pd clusters present on the graphene surface at high coverages of Pd. Panels (b) - (d) of Figure 2 show series of IRAS spectra of CO on several $\mathrm{Pd} / \mathrm{GR} / \mathrm{Ru}(0001)$ systems with different coverages of palladium under $\mathrm{CO}$ atmosphere $\left(1.0 \times 10^{-}\right.$ ${ }^{6}$ Torr) obtained at $300 \mathrm{~K}$ sample temperature. Over various coverage of Pd (1.0 - 5.0 MLE), IRAS spectra of adsorbed CO show very similar patterns as those of 0.25 MLE of $\mathrm{Pd} / \mathrm{GR} / \mathrm{Ru}(0001)$. At annealing temperatures up to $\sim 1000 \mathrm{~K}$, CO vibrational feature appears, but the intensity decreases with increasing annealing temperature. Between 1100 and $1300 \mathrm{~K}$, the $\mathrm{CO}$ vibrational feature completely disappears even under $\mathrm{CO}$ atmosphere. If the intercalation 
and subsequent desorption of palladium would cause any damage or loss to graphene layer we should be able to monitor it by the characteristic vibrational features of adsorbed $\mathrm{CO}$ on the exposed $\mathrm{Ru}$ metal sites. However, no evidence of any adsorbed $\mathrm{CO}$ is seen on the high temperature $(>1100 \mathrm{~K})$ annealed $\mathrm{Pd} / \mathrm{GR} / \mathrm{Ru}(0001)$ samples, not even for the highest Pd covered one (5.0 MLE). This observation clearly indicates the high structural stability of the graphene layer during the thermal stability study of $\mathrm{Pd}$ overlayers. The graphene layer remains intact without any physical damage during intercalation and subsequent desorption of the Pd overlayer. This result is corresponding to the exchange mechanism of intercalation. Jin et al. [9] reported that $\mathrm{Ni}$ adatoms anchoring on graphene layer may produce transient atomic-scale defects at the graphene lattice by the strong interaction between $\mathrm{Ni}$ and carbon and $\mathrm{Ni}$ diffused through this transient defects. After Ni adatoms pass though the graphene layer and intercalated, the transient defects are healed by itself and a perfect graphene surface is reformed [9]. Our IRAS results after intercalation of Pd under graphene and after the partial desorption of Pd do not show any $\mathrm{CO}$ vibrational features. If there is any extended defect or additional defect formed during the intercalation and/or desorption of $\mathrm{Pd}, \mathrm{CO}$ vibrational feature should be immediately appeared. This observation apparently shows that Pd pass through the graphene layer without any damage during the intercalation and the desorption from substrate. The differences in the IRAS spectra for samples with different Pd coverages arise primarily from the different initial sizes of the metal clusters formed as the Pd coverage was varied from 0.25 to 5.0 MLE. At the lowest Pd coverage of 0.25 MLE, almost exclusively one type of bridge-bound CO is present, which is represented by the IR feature centered at $1975 \mathrm{~cm}^{-1}$. Libuda et al. [27] previously reported that palladium clusters supported on alumina have $\operatorname{Pd}(111)$ and $\operatorname{Pd}(100)$ facets and they assigned the 1930 and $1840 \mathrm{~cm}^{-1}$ features to $\mathrm{CO}$ adsorbed on $\operatorname{Pd}(111)$, the $1960 \mathrm{~cm}^{-1}$ band to a superposition 
of bridge-bound $\mathrm{CO}$ on $\mathrm{Pd}(100)$ facets and $\mathrm{CO}$ adsorbed on defect sites, such as particle edges or steps, and the $2080 \mathrm{~cm}^{-1}$ feature is $\mathrm{CO}$ adsorbed in on-top geometry. As shown in Figure 2, every series of IR spectra shows one prominent feature at $1975-1990 \mathrm{~cm}^{-1}$ with low intensity features at $\sim 2085$ and $\sim 1930 \mathrm{~cm}^{-1}$. It clearly demonstrates the presence of small threedimensional palladium clusters on the graphene layer and this morphology maintained until palladium is intercalated. The decrease in IR intensity as a function of annealing temperature substantiates the agglomeration of Pd clusters as the annealing temperature increases. The Pd particles at low coverage ( 0.25 MLE) are small, and no dominant, large crystallographic planes ((100) and (111)) have developed. With increasing Pd coverage three trends are seen on the series of IR spectra: 1 . The total integrated intensity of the IR features of adsorbed CO increases with Pd loading; 2. The position of the above discussed IR feature characteristic of bridge-bound CO on small Pd clusters gradually shifts to higher wavenumbers $\left(1975 \mathrm{~cm}^{-1}\right.$ at 0.25 MLE and $\sim 1990 \mathrm{~cm}^{-1}$ for the 5 MLE sample); and 3. Two new bands develope: one at a lower frequency (characteristic of another type of bridge-bond CO), and one at higher frequency (representing linearly adsorbed CO). The overall intensity increase of the IR features of adsorbed $\mathrm{CO}$ is in concert with the increase in available adsorption sites as the amount of Pd deposited increases. The changes in the IR peak positions and the development of two new absorption features as the amount of Pd deposited increases are direct consequences in the change of the Pd clusters (both sizes and shapes). As the amount of Pd deposited increases large Pd clusters with different crystallographic planes form, and the adsorbed CO probes these different sites.

\section{Conclusions}


The thermal behaviors of $\mathrm{Pd}$ overlayers at various coverages on graphene/Ru(0001) have been investigated as a function of annealing temperature using surface spectroscopic techniques. For all Pd coverages studied three distinct processes were identified: annealing the room temperature-deposited metal overlayers to $\sim 900 \mathrm{~K}$ resulted in the agglomeration of Pd clusters. In the temperature range of $\sim 900-1100 \mathrm{~K} \mathrm{Pd}$ intercalated between the graphene layer and the $\mathrm{Ru}(0001)$ substrate. Above $1100 \mathrm{~K}$ Pd begins to desorb, and by $1400 \mathrm{~K}$ all the Pd overlayer was removed by desorption, while the graphene layer dissolved into the metal substrate. These processes were clearly evidenced from both the variation in the intensities of the metal XPS signals and the IRAS spectra of adsorbed CO during annealing in vacuum. The IRAS feature of bridge-bound $\mathrm{CO}$ on Pd/graphene blue-shifted with increasing Pd coverage demonstrating that small and highly stepped clusters are formed during the deposition of Pd at room temperature. Due to the weak interaction between Pd and graphene agglomeration of Pd clusters occurred at low annealing temperatures. However, higher temperature annealing (but below the desorption temperature of $\mathrm{Pd}$ ) results in the intercalation of palladium between graphene and ruthenium, and at even higher temperature the intercalated Pd desorbs and the graphene layer is dissolved in $\mathrm{Ru}$ substrate. Interestingly, no noticeable structural changes were observed in the graphene layer either during intercalation or Pd desorption.

\section{Acknowledgments}

We gratefully acknowledge the US Department of Energy (DOE), Office of Science, Office of Basic Energy Sciences, Chemical Sciences, Geosciences, and Biosciences Division for the support of this work. The research described in this article was performed at the Environmental Molecular Sciences Laboratory (EMSL), a national scientific user facility 
sponsored by the DOE Office of Biological and Environmental Research and located at Pacific Northwest National Laboratory (PNNL). PNNL is operated for the US DOE by Battelle Memorial Institute under contract number DE-AC05-76RL01830. This work was also supported by the Sungshin Women's University Research Grant of 2014. 


\section{References}

[1] M. Batzill, Surf. Sci. Rep. 67 (2012) 83-115.

[2] A. Dahal, M. Batzill, Nanoscale 6 (2014) 2548-2562.

[3] K. Gotterbarm, C. Bronnbauer, U. Bauer, C. Papp, H.P. Steinruck, J. Phys. Chem. C 118 (2014) 25097-25103.

[4] K. Gotterbarm, C. Steiner, C. Bronnbauer, U. Bauer, H.P. Steinruck, S. Maier, C. Papp, J. Phys. Chem. C 118 (2014) 15934-15939.

[5] Q. Liao, H.J. Zhang, K. Wu, H.Y. Li, S.N. Bao, P. He, Surf. Rev. Lett. 19 (2012) 5.

[6] M. Liu, R. Zhang, W. Chen, Chemical Reviews 114 (2014) 5117-5160.

[7] C. Tan, X. Huang, H. Zhang, Materials Today 16 (2013) 29-36.

[8] Y. Cui, J.F. Gao, L. Jin, J.J. Zhao, D.L. Tan, Q. Fu, X.H. Bao, Nano Res. 5 (2012) 352-360.

[9] L. Jin, Q. Fu, Y. Yang, X.H. Bao, Surf. Sci. 617 (2013) 81-86.

[10] C.-W. Yi, J.H. Kwak, C.H.F. Peden, C. Wang, J. Szanyi, J. Phys. Chem. C 111 (2007) 14942-14944.

[11] C.-W. Yi, J.H. Kwak, J. Szanyi, J. Phys. Chem. C 111 (2007) 15299-15305.

[12] C.-W. Yi, J. Szanyi, J. Phys. Chem. C 113 (2009) 716-723.

[13] C.-W. Yi, J. Szanyi, J. Phys. Chem. C 113 (2009) 2134-2140.

[14] S. Axnanda, K.D. Cummins, T. He, D.W. Goodman, M.P. Soriaga, ChemPhysChem 11 (2010) 14681475.

[15] C.W. Yi, K. Luo, T. Wei, D.W. Goodman, J. Phys. Chem. B 109 (2005) 18535-18540.

[16] S.R. Wang, Y. Zhang, N. Abidi, L. Cabrales, Langmuir 25 (2009) 11078-11081.

[17] Z.H. Zhou, F. Gao, D.W. Goodman, Surf. Sci. 604 (2010) 1071-L1038.

[18] A.T. N'Diaye, S. Bleikamp, P.J. Feibelman, T. Michely, Physical Review Letters 97 (2006).

[19] A.T. N'Diaye, T. Gerber, C. Busse, J. Myslivecek, J. Coraux, T. Michely, New Journal of Physics 11 (2009).

[20] H. Unterhalt, G. Rupprechter, H.-J. Freund, J. Phys. Chem. B 106 (2001) 356-367.

[21] I.V. Yudanov, et al., J. Phys. Chem. B 107 (2002) 255-264.

[22] K. Gotterbarm, W. Zhao, O. Hofert, C. Gleichweit, C. Papp, H.P. Steinruck, Phys. Chem. Chem. Phys. 15 (2013) 19625-19631.

[23] D.E. Pierce, R.P. Burns, K.A. Gabriel, Thin Solid Films 206 (1991) 340-344.

[24] J. Storm, R.M. Lambert, N. Memmel, J. Onsgaard, E. Taglauer, Surf. Sci. 436 (1999) 259-268.

[25] H. Hartmann, T. Diemant, J. Bansmann, R.J. Behm, Surf. Sci. 603 (2009) 1456-1466.

[26] H. Pfnür, D. Menzel, F.M. Hoffmann, A. Ortega, A.M. Bradshaw, Surf. Sci. 93 (1980) 431-452.

[27] J. Libuda, H.J. Freund, Surf. Sci. Rep. 57 (2005) 157-298. 


\section{Figure Captions}

Figure 1. Normalized XPS intensities of $\mathrm{Pd}, \mathrm{Ru}$, and the ratio of $\mathrm{Pd} / \mathrm{Ru}$ for (a) $0.25 \mathrm{MLE}$, (b) 1.0 MLE, (c) 2.0 MLE, and (d) 5.0 MLE Pd/graphene/Ru(0001) as a function of annealing temperature. The palladium was deposited at room temperature.

Figure 2. IRAS spectra of adsorbed CO on (a) 0.25 MLE, (b) 1.0 MLE, (c) 2.0 MLE, and (d) 5.0 $\mathrm{MLE} \mathrm{Pd} /$ graphene/Ru(0001) systems at a CO pressure $\left(1.0 \times 10^{-6} \mathrm{Torr}\right)$ as a function of annealing temperature. All the IR spectra were taken at room temperature.

Figure S1. Calibration curve for Pd deposition: Pd/Ru XPS signal intensity ratio as a function of deposition time at a constant rate of metal evaporation.

Figure S2. LEED pattern for pristine graphene at $70.4 \mathrm{eV}$ (a) and $15.4 \mathrm{eV}$ (b).

Figure S3. Series of XP spectra of Pd 3d (panel a) and Ru 3d (panel b) collected during the stepwise annealing of a $1 \mathrm{MLE} \mathrm{Pd} / \mathrm{GR} / \mathrm{Ru}(0001)$ sample. 


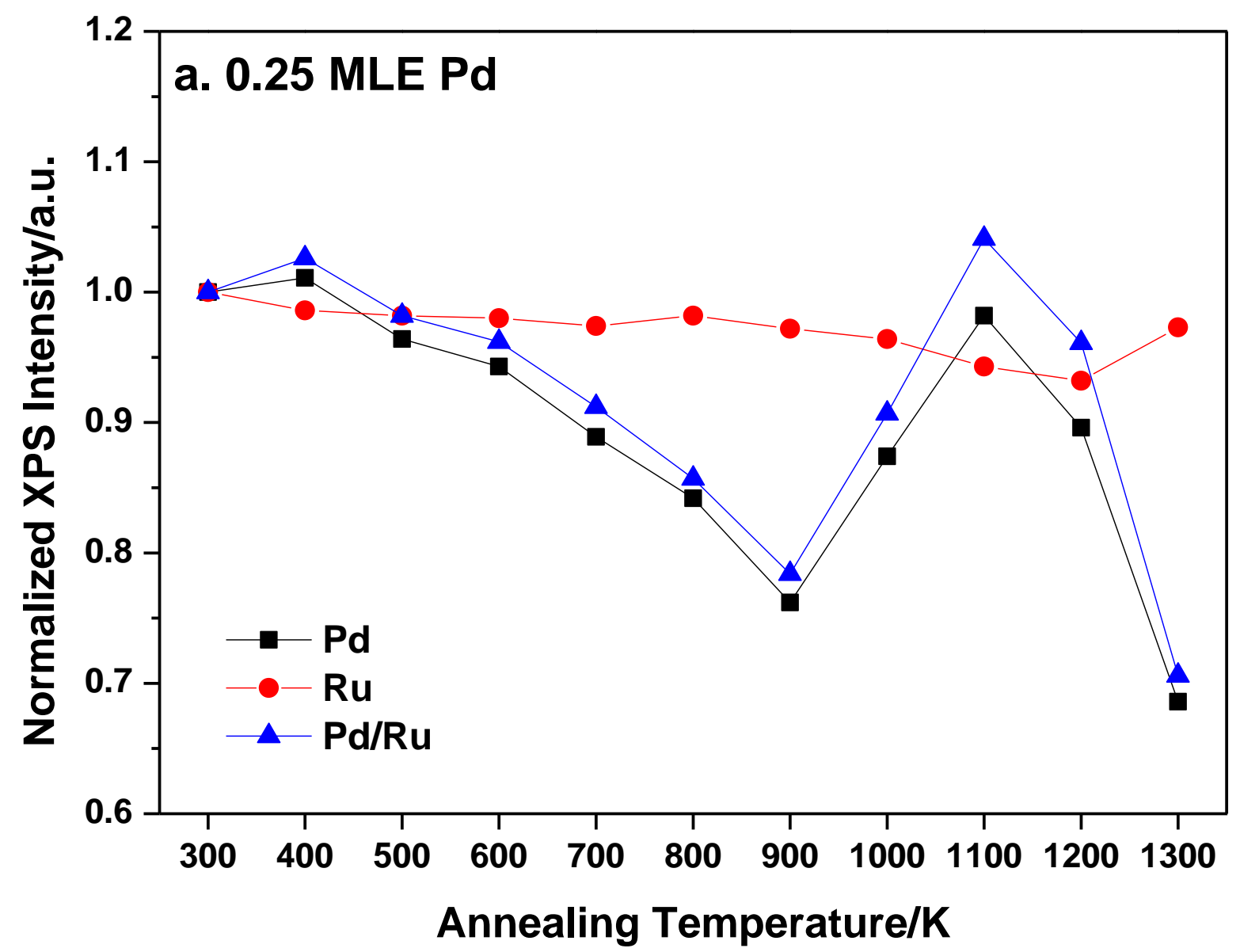

Figure 1 (a) Yi et.al 


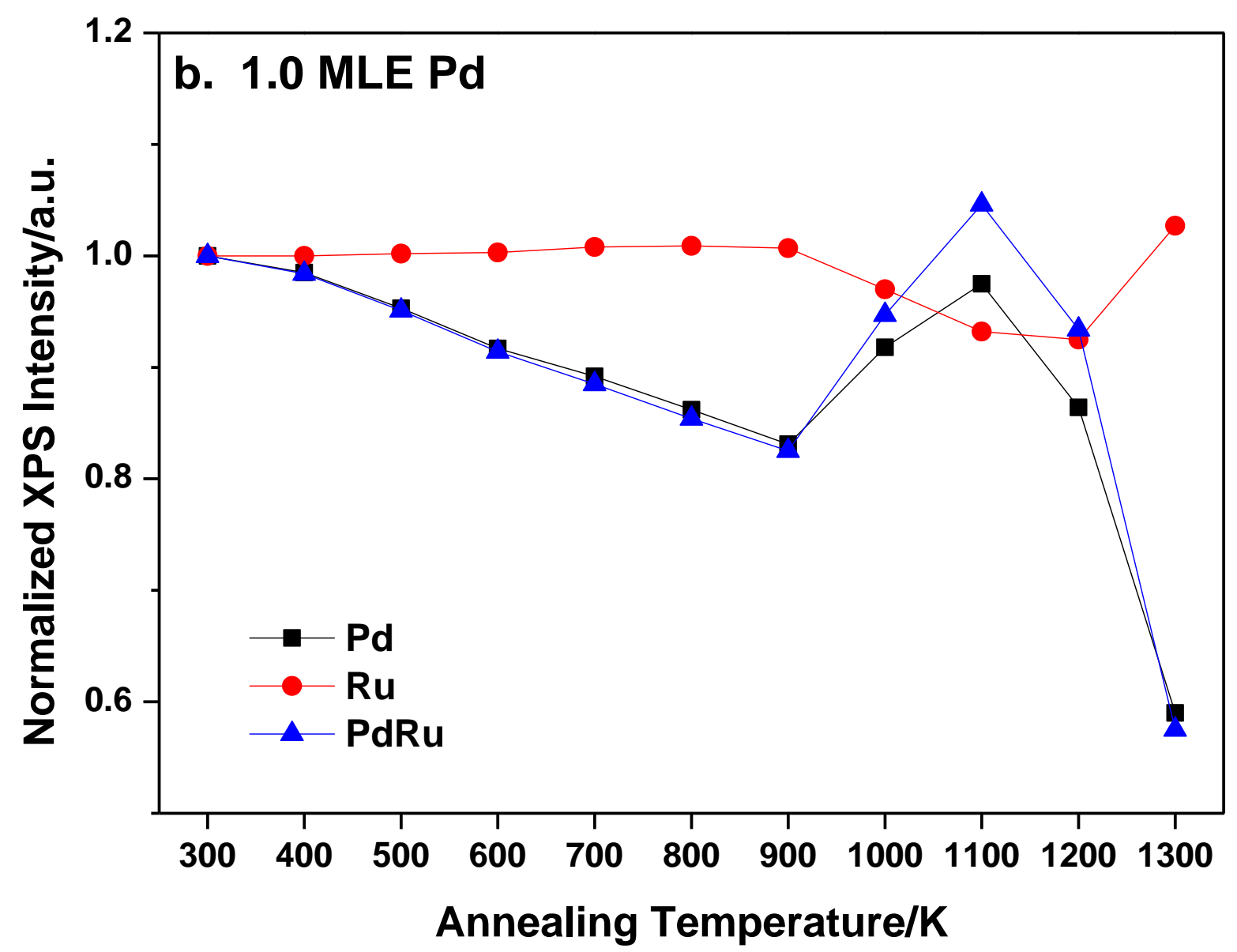

Figure 1 (b) Yi et.al 


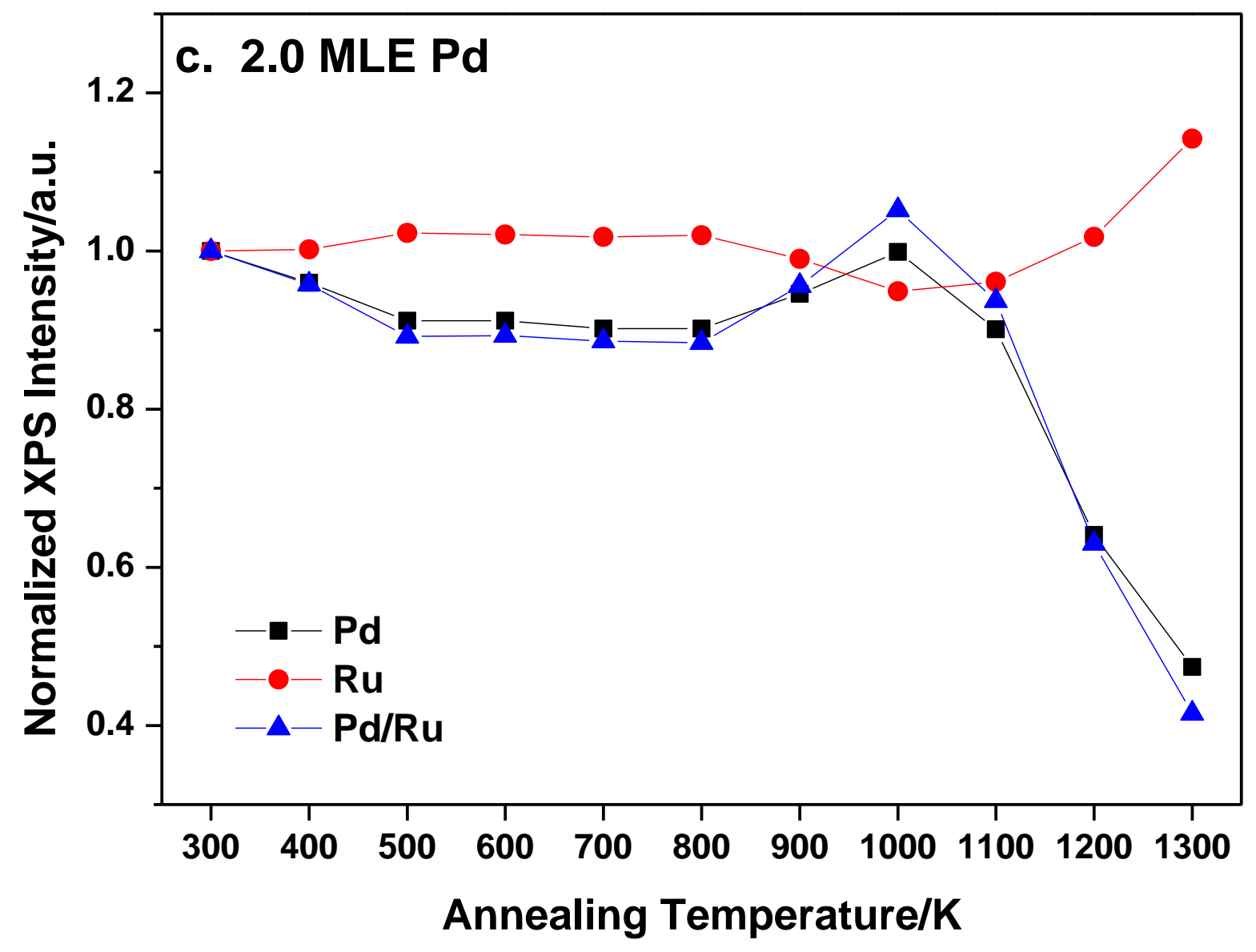

Figure 1 (c) Yi et.al 


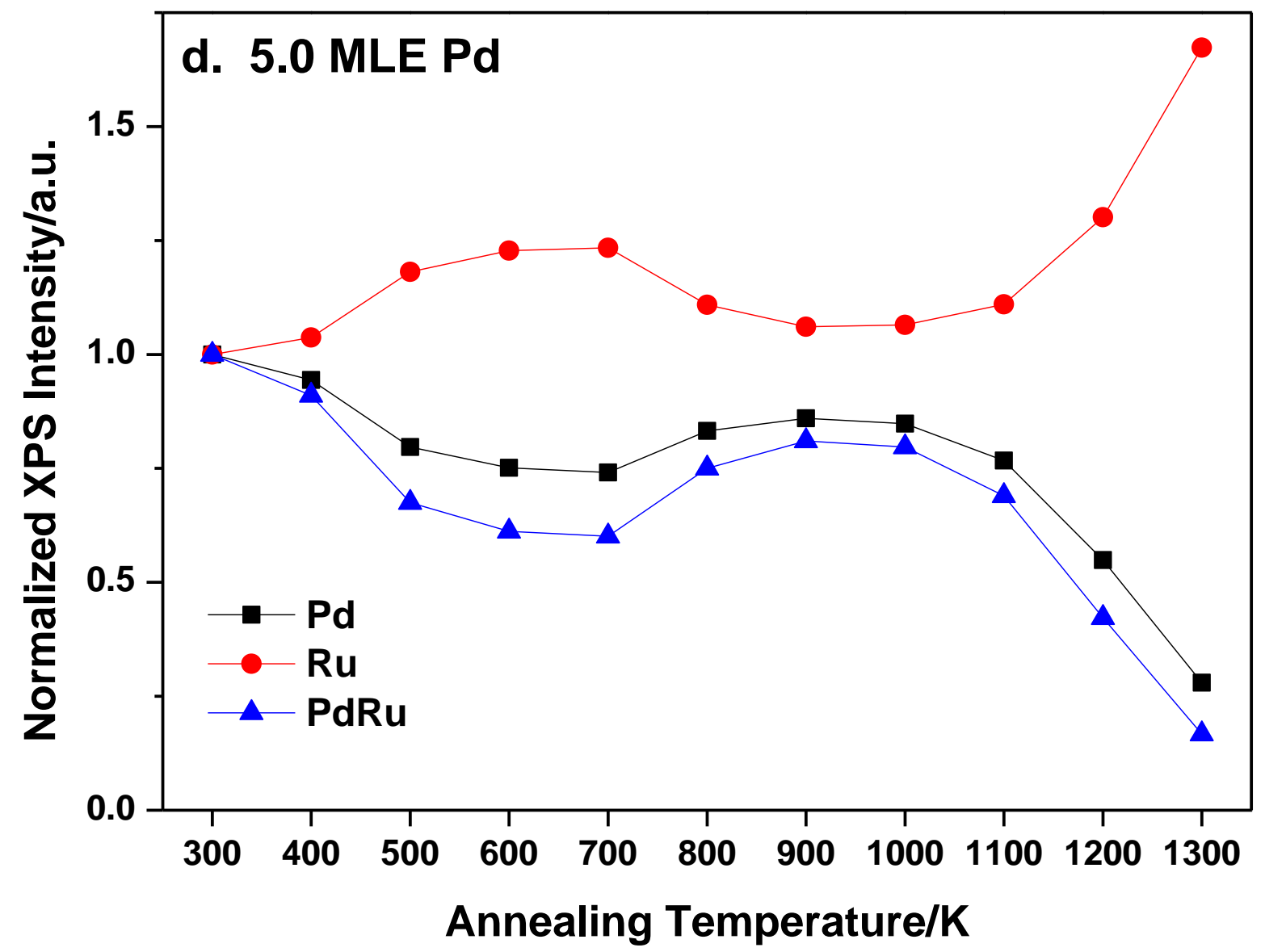

Figure 1 (d) Yi et.al 


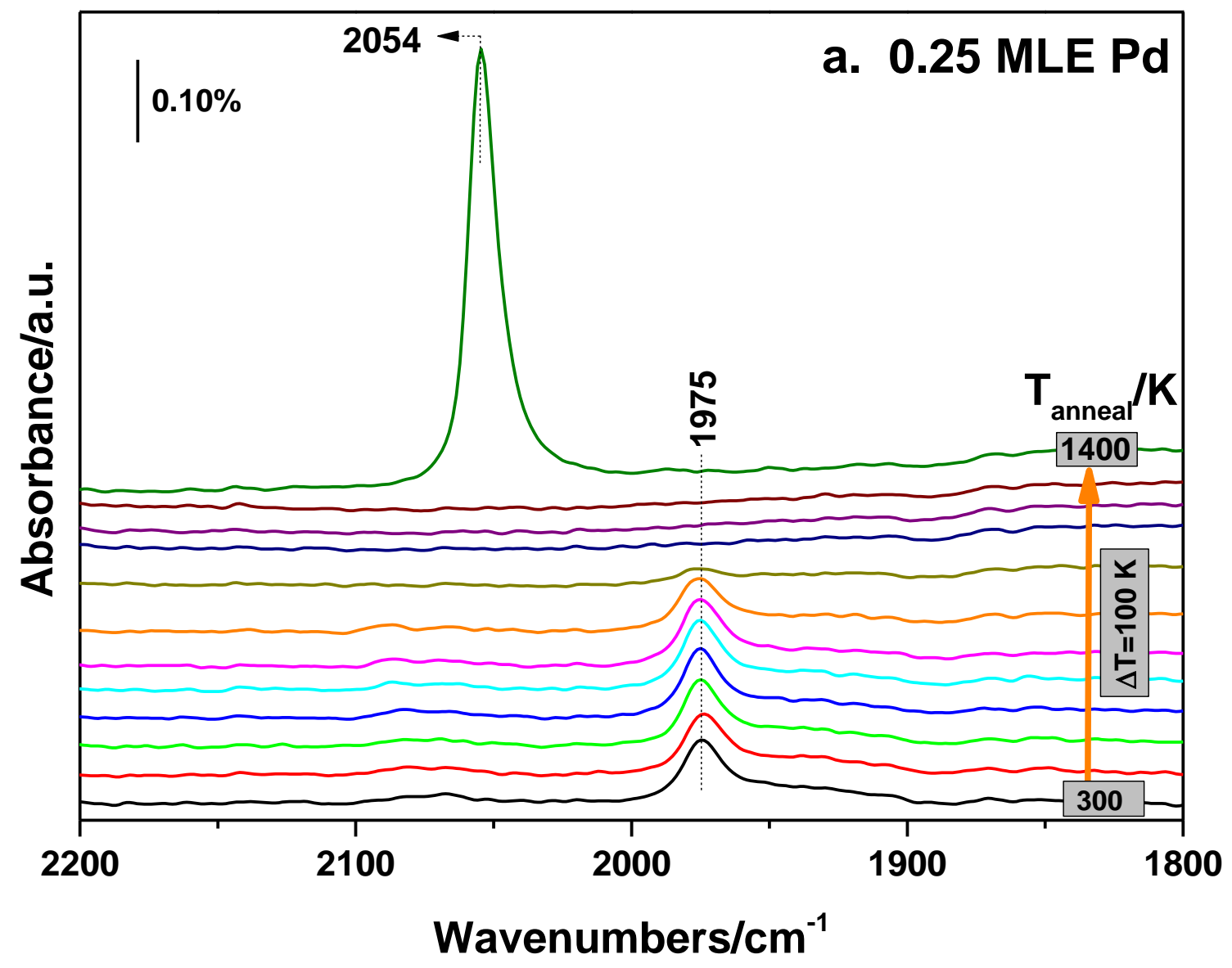

Figure 2 (a) Yi et.al 


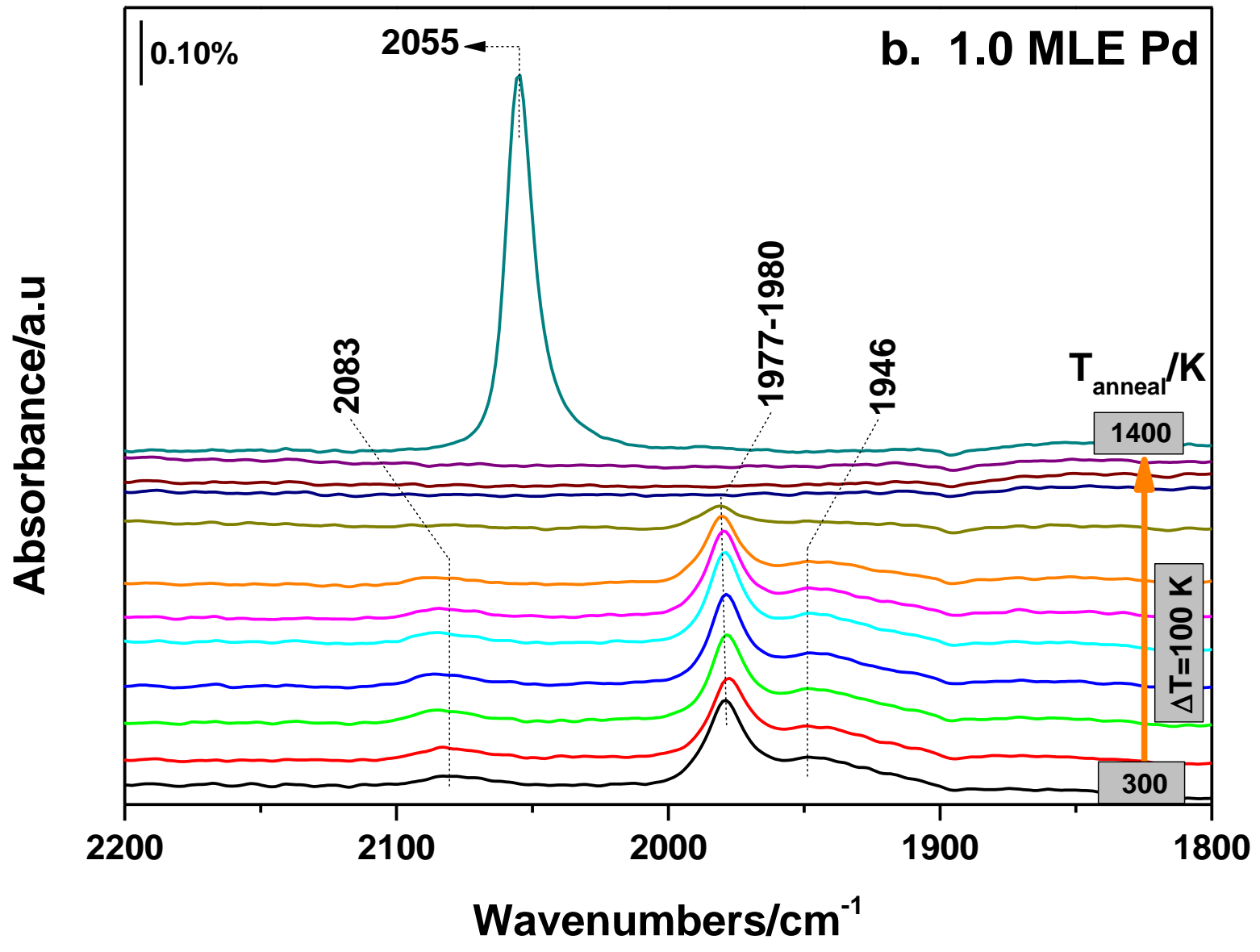

Figure 2 (b) Yi et.al 


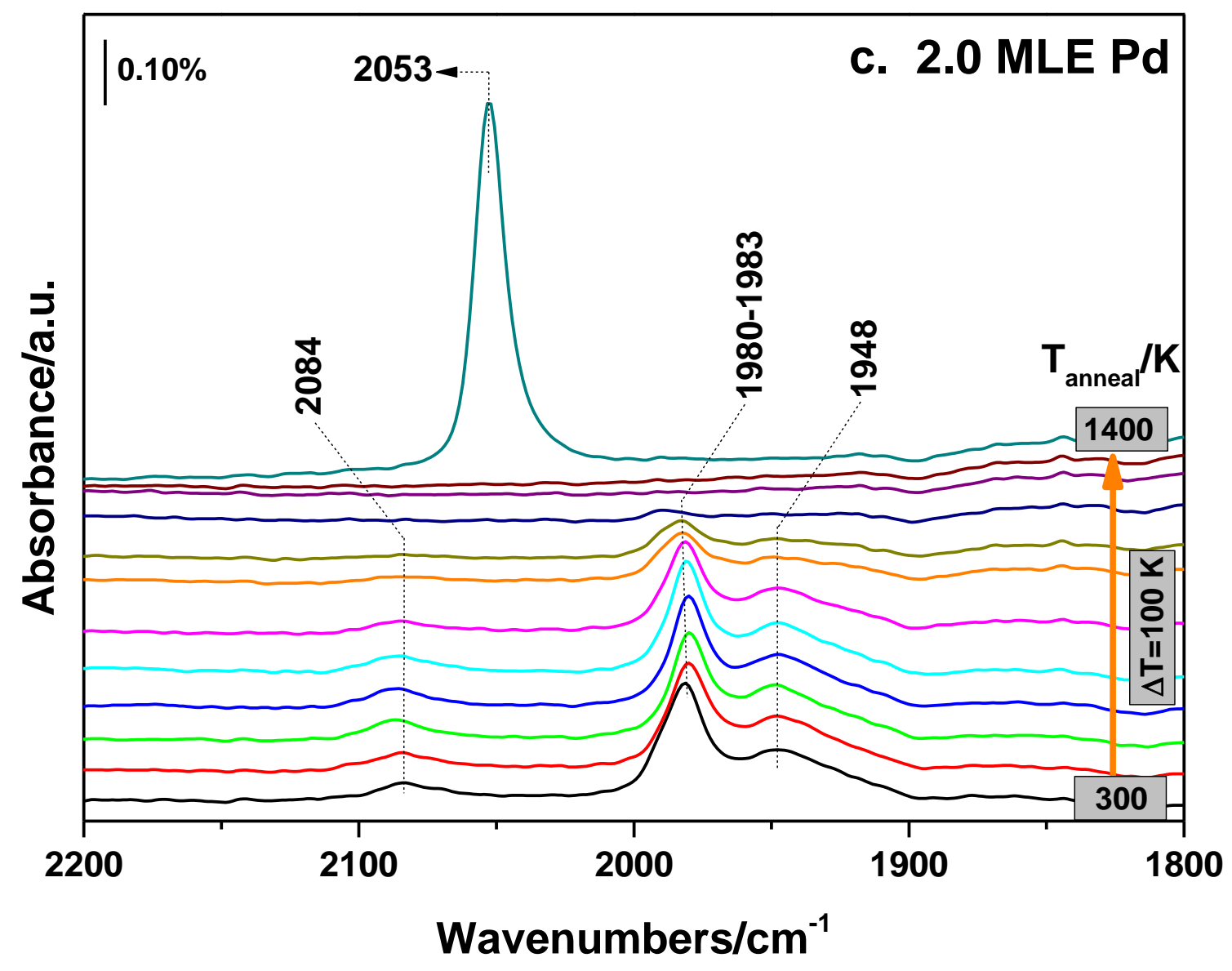

Figure 2 (c) Yi et.al 


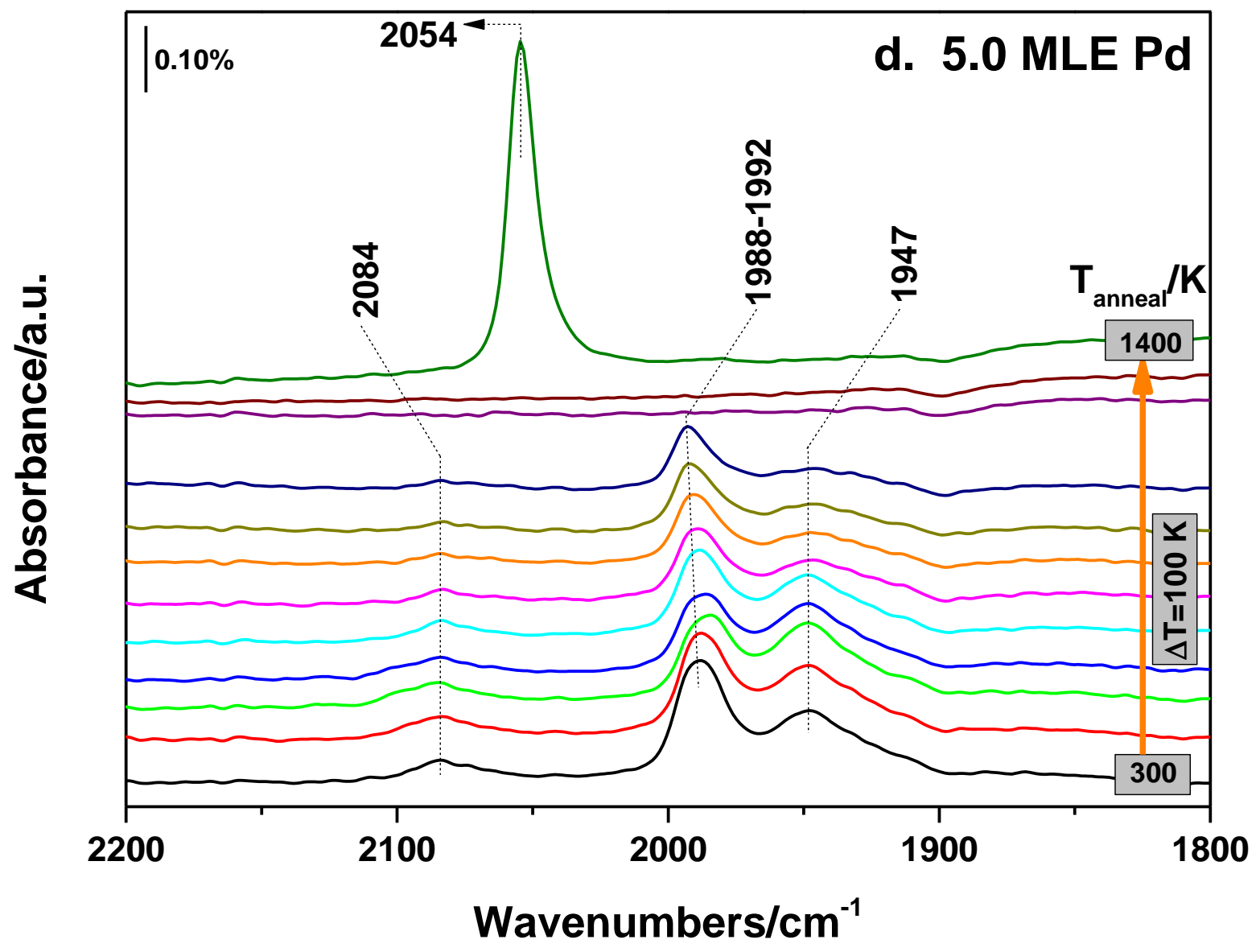

Figure 2 (d) Yi et.al 


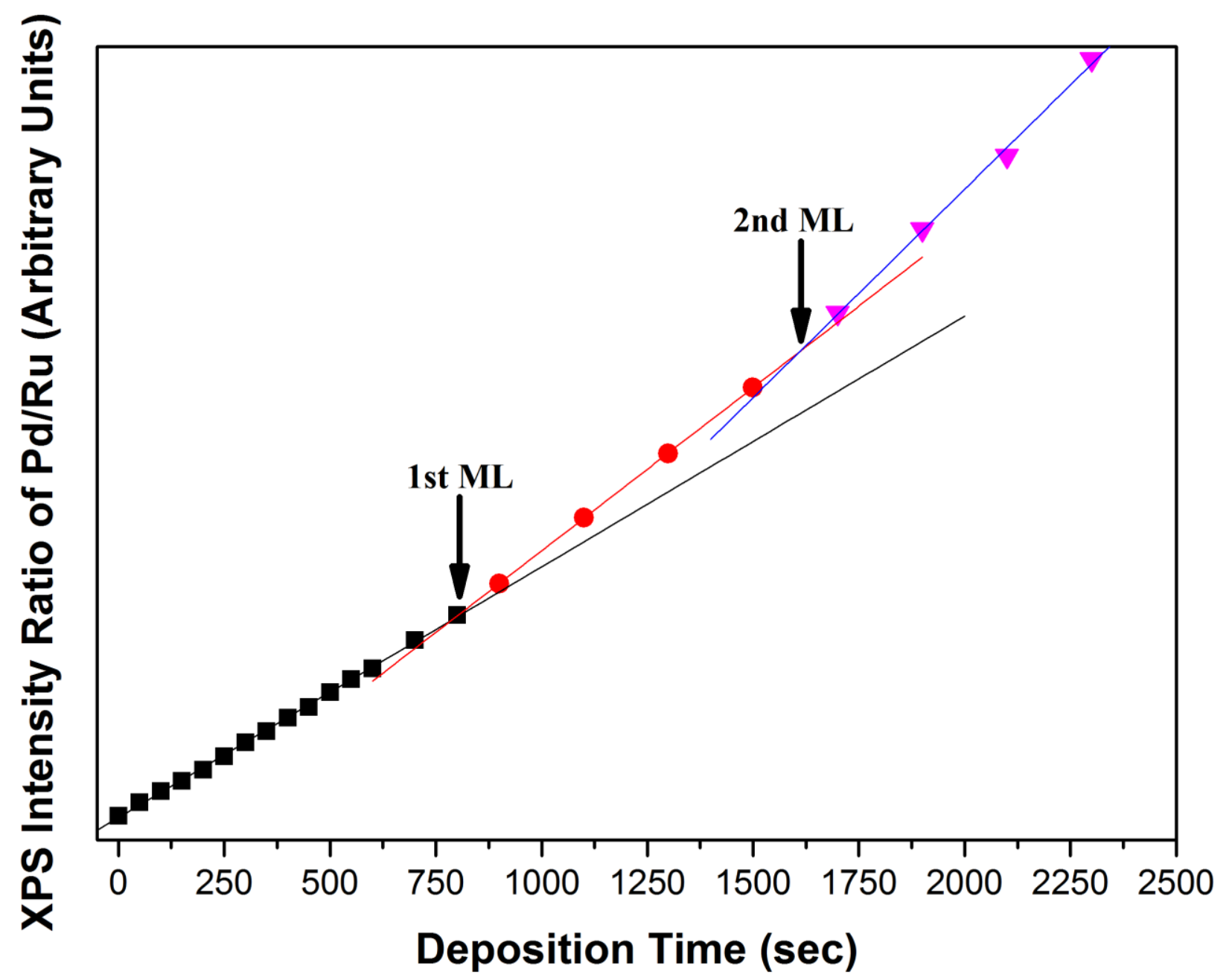

Figure S1 Yi et.al 


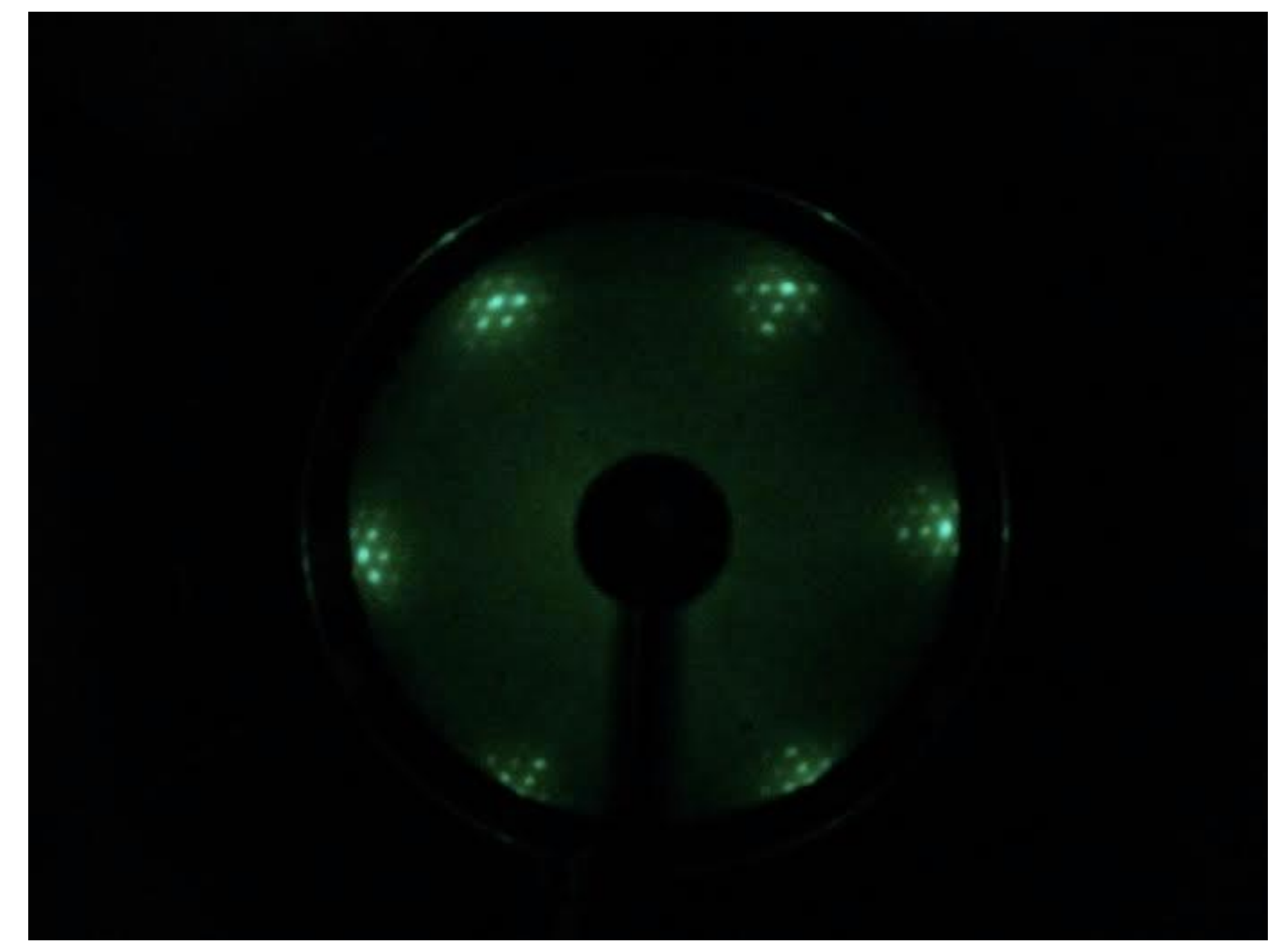

Figure S2 (a) Yi et.al 
Figure S2 (b) Yi et.al 


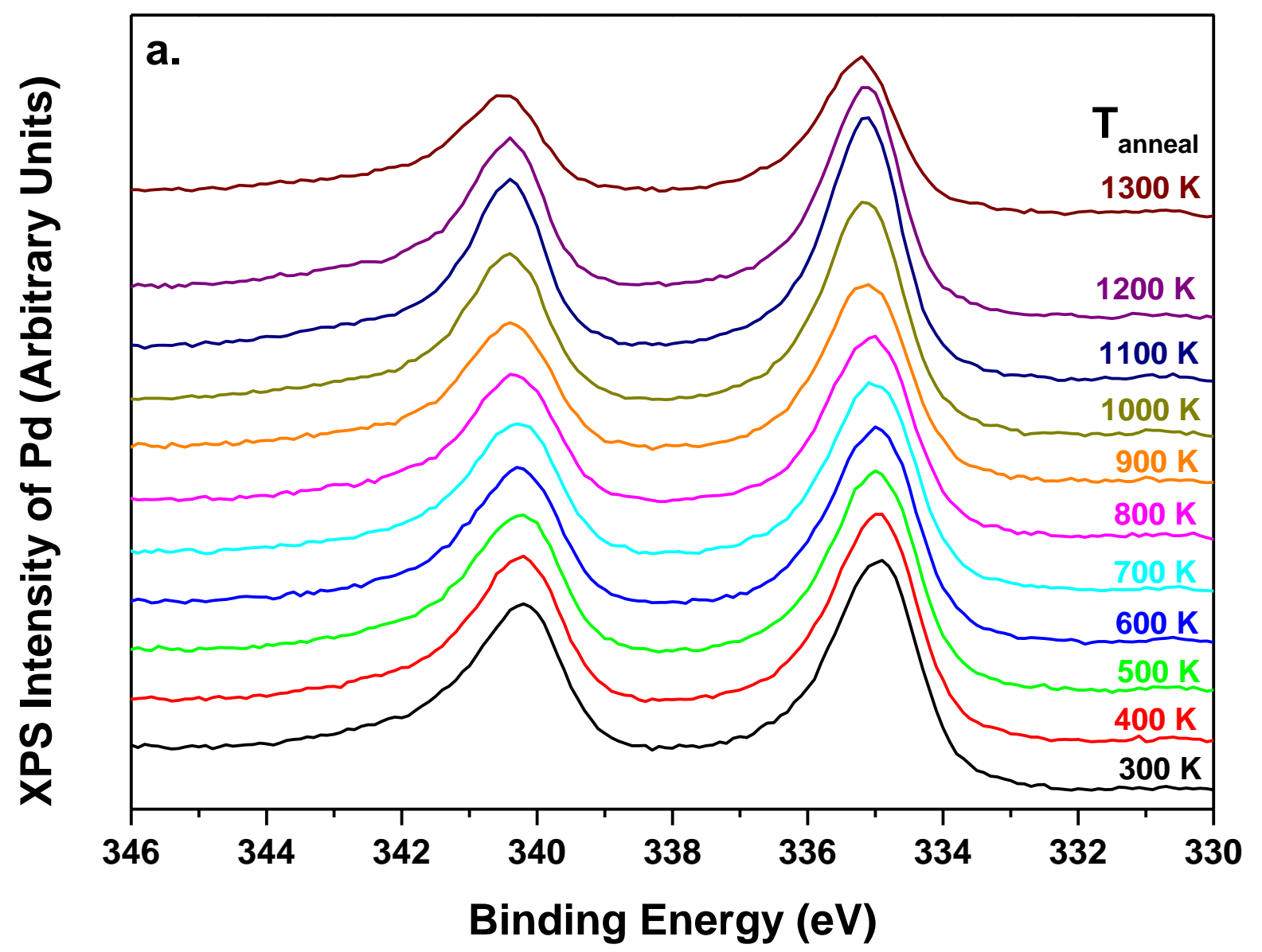

Figure S3 (a) Yi et.al 


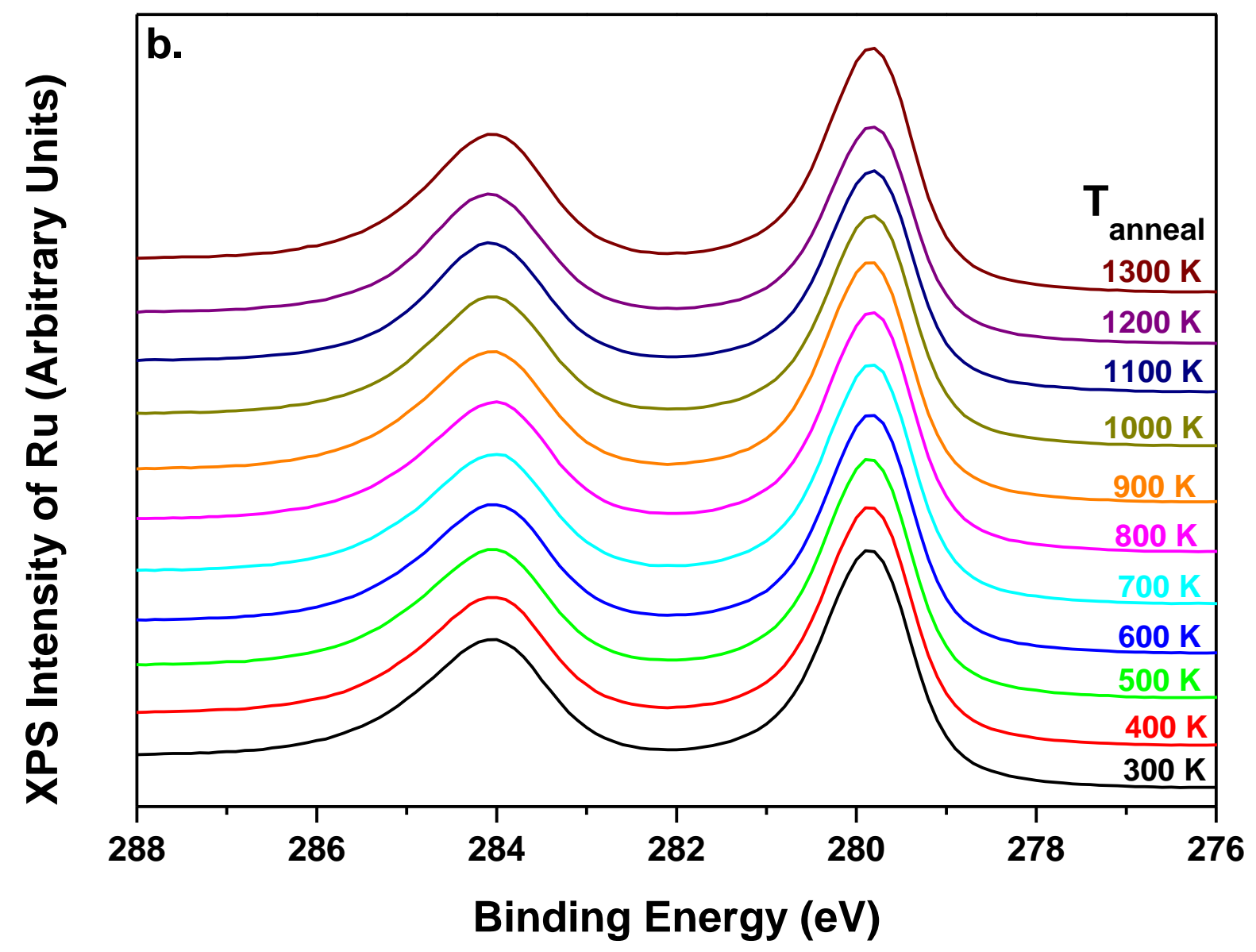

Figure S3 (b) Yi et.al 



\title{
The Thermal Behavior of Pd on Graphene/Ru(0001)
}

\author{
Cheol-Woo Yi ${ }^{\mathrm{a}, \mathrm{b}}$ and János Szanyi ${ }^{*} \mathrm{~b}$ \\ ${ }^{a}$ Department of Chemistry and Institute of Basic Science, Sungshin Women's University, Seoul \\ 142-732, South Korea \\ ${ }^{b}$ Institute for Integrated Catalysis, Pacific Northwest National Laboratory, P.O. Box 999, MSIN: \\ K8-87, Richland, WA, USA
}

The thermal behavior of various amounts of palladium deposited onto graphene/Ru(0001) at room temperature was investigated by x-ray photoelectron spectroscopy (XPS) and infrared reflection absorption spectroscopy (IRAS) of adsorbed CO. IRAS spectra of adsorbed CO on palladium deposited onto the graphene layer reveal the formation of defect-rich, highly-stepped and/or nanoparticle-typed palladium clusters. Annealing to temperatures below $~ 900 \mathrm{~K}$, palladium clusters mainly experience agglomeration on the graphene layer, but at higher temperatures intercalation of palladium between the graphene layer and Ru substrate takes place. Eventually, palladium completely desorbs and the graphene layer dissolves into the ruthenium substrate at $1400 \mathrm{~K}$. Even though the annealing induces the intercalation and desorption of palladium, the topmost graphene layer stays intact without any physical damage at and below $1300 \mathrm{~K}$.

\footnotetext{
* To whom correspondence should be addressed. E-mail: janos.szanyi@pnnl.gov
} 


\section{Introduction}

Graphene is a single atomic layer consisting of $\mathrm{sp}^{2}$-hybridized carbon. It is expected to be applied in many fields due to its remarkable electronics properties [1]. Because of its importance, the interfaces between graphene and transition metals have been investigated extensively $[1,2]$. Moreover, the nano-composite materials consisting of graphene and transition metal(s) are attracting a great attention for numerous technological applications in the field of electronics, material science, catalysis and so on [1,2]. The interfaces between graphene and transition metals have been recently investigated [1-5]. Among them, palladium-graphene composite is employed in various chemical sensors and catalytic reactions [6,7]. Investigations on well-defined model systems can greatly contribute to the understanding of the properties of metal overlayers on graphene. The epitaxial growth of high-quality graphene has recently been investigated on various metal and inorganic material surfaces as substrates $[2,8]$. Despite the great importance of metal particles on graphene substrates, fundamental studies of the physical and chemical properties of palladium clusters on graphene are still needed. Two distinct intercalation mechanisms of metal under the graphene layer were proposed by the strength of interaction between intercalant and carbon [9]. It is believed that the metal, which weakly interact with carbon, such as $\mathrm{Pb}$, is intercalated through extended defects (open edge and/or domain boundaries), but the metal strongly interacted with carbon via exchange mechanism [9]. The intercalation via exchange mechanism needs higher temperature than that through extended defects. In this study, palladium was deposited onto graphene/Ru(0001) surfaces at room temperature, and the thermal behaviors of palladium clusters have been investigated with x-ray photoelectron spectroscopy (XPS), low energy electron diffraction (LEED), and infrared reflection absorption spectroscopy (IRAS) of adsorbed CO. Under low temperature (up to 800 
K) annealing in vacuum, palladium clusters mainly experience agglomeration on the graphene layer. However, further annealing at higher temperature $(\sim 900-1100 \mathrm{~K})$ causes to the intercalation of palladium between the graphene layer and Ru substrate, and then Pd clusters desorb from the substrate at temperatures $>1100 \mathrm{~K}$. Even though the annealing induces the intercalation of palladium between graphene and ruthenium substrate and desorption of palladium, the topmost graphene layer remained intact. This observation is a good agreement with hypothetical exchange mechanism of intercalation.

\section{Experimental}

The experimental setup and the data acquisition procedures have been discussed in detail elsewhere [10-13]. All the experiments were performed in an ultrahigh vacuum (UHV) surface analysis chamber equipped with x-ray photoelectron spectroscopy, low-energy electron diffraction, and quadrupole mass spectrometry, and connected to an elevated-pressure cell equipped with $\mathrm{KBr}$ windows for infrared reflection absorption spectroscopy measurements. The base pressure in both chambers was $\sim 1 \times 10^{-10}$ Torr. The $\mathrm{Ru}(0001)$ single crystal used in these experiments (Princeton Scientific Corp., diameter $=10 \mathrm{~mm}$ and thickness $=1 \mathrm{~mm}$ ) was spotwelded onto a U-shaped Ta wire (0.030 in. diameter). The sample temperature was measured by a C-type thermocouple spot-welded to the backside of the single crystal. The $\mathrm{Ru}(0001)$ single crystal was cleaned by repeated cycles of Ar ion sputtering, oxidation at $1000 \mathrm{~K}$ and annealing up to $1900 \mathrm{~K}$ in UHV, and the cleanliness and order of the surface were verified with XPS and LEED, respectively. The high quality epitaxial graphene layer is prepared by pyrolysis of ethylene on $\mathrm{Ru}(0001)$ surface. The $\mathrm{Ru}$ was annealed at $1400 \mathrm{~K}$ for $300 \mathrm{sec}$ and then the sample was rapidly cooled down under ethylene atmosphere $\left(1 \times 10^{-6} \mathrm{Torr}\right)$. The quality of graphene 
layer was monitored with LEED and IRAS with the adsorption of carbon monoxide. Pd was deposited at room temperature onto the graphene layer supported on $\mathrm{Ru}(0001)$ using a resistively heated palladium wire (Alfa Aesar; diameter $=0.25 \mathrm{~mm} ; 99.99 \%$ ). The palladium coverage was calibrated with respect to clean $\mathrm{Ru}$ by the break point of XPS peak ratio $(\mathrm{Pd} / \mathrm{Ru})$ as shown in Figure S1 [14,15]. Infrared spectra were collected at $4 \mathrm{~cm}^{-1}$ resolution using a grazing angle of approximately $85^{\circ}$ to the surface normal. All the IR spectra collected were referenced to a background spectrum, which is acquired from the sample prior to gas adsorption, and taken under $\mathrm{CO}$ atmosphere $\left(1 \times 10^{-6}\right.$ Torr $)$ at saturation $\mathrm{CO}$ coverage.

\section{Results and discussion}

The quality of the prepared graphene layer was monitored by XPS, LEED, and IRAS.

The infrared spectrum obtained from the graphene layer after $\mathrm{CO}$ exposure $\left(1 \times 10^{-6}\right.$ Torr of $\left.\mathrm{CO}\right)$ at room temperature showed no $\mathrm{CO}$ vibrational features, indicating full and continuous coverage of carbon on the Ru surface (i.e., no exposed $\mathrm{Ru}$ atoms were present). Carbon monoxide is one of the best probe molecules to identify defect sites on the surface. Due to the strong interaction of $\mathrm{CO}$ with $\mathrm{Ru}$ even at room temperature, $\mathrm{CO}$ vibrational features should appear once the graphene layer is defective and/or Ru sites are exposed through the "holes" in the graphene. Hence, the sharp LEED pattern (shown in Figure S2 (a) and (b)) and the absence of any CO vibrational feature in the IRAS spectrum unambiguously show that the graphene film is of good quality and fully covers the ruthenium substrate. After the desired amount of Pd was deposited onto the graphene (GR) layer at room temperature, the sample was annealed step-wise (in $100 \mathrm{~K}$ intervals) and changes in the Pd/GR/Ru(0001) systems were monitored by both XPS and IRAS of adsorbed CO. The samples were kept at each annealing temperature for 5 minutes. Figures 1 
(a) and 2 (a) show the normalized XPS intensities of $\mathrm{Pd}, \mathrm{Ru}$, and $\mathrm{Pd} / \mathrm{Ru}$ in UHV and infrared spectra during $\mathrm{CO}$ exposure $\left(1.0 \times 10^{-6}\right.$ Torr of $\left.\mathrm{CO}\right)$ for 0.25 monolayer equivalent (MLE) $\mathrm{Pd} / \mathrm{GR} / \mathrm{Ru}(0001)$ system as a function of annealing temperature, respectively. In the temperature range of $300-900 \mathrm{~K}$, the XPS intensity of Pd gradually decreases with increasing annealing temperature. This phenomenon is attributed to the agglomeration of Pd clusters deposited onto the graphene layer as the sample temperature was increased. The surface free energy of Pd is reported to be $2.043 \mathrm{~J} / \mathrm{m}^{2}$ [15] which is significantly higher than that of graphene, $4.67 \times 10^{-2}$ $\mathrm{J} / \mathrm{m}^{2}[16]$. The deposited Pd forms three-dimensional clusters on the graphene layer due to the difference of surface free energies and the sufficient mobility of Pd atoms [17]. Moreover, N'Diaye et al. [18,19] and Batzill [1] reported that the formation and stability of metal cluster on graphene moiré structure depend on several parameters such as lattice constant and cohesive energies of the deposited materials as well as the interaction between the metal and the graphene. N'Diaye et al. observed the high stability of $\operatorname{Ir} /$ graphene/Ir(111) and suggested the extra stabilization owing to the formation of strong metal-carbon bond via $\mathrm{sp}^{2} \rightarrow \mathrm{sp}^{3}$ re-hybridization of carbon below the metal cluster $[1,18,19]$. Hence, the dissociation energy $(436 \mathrm{~kJ} / \mathrm{mol})$ of $\mathrm{Pd}-$ $\mathrm{C}$ bond is relatively lower than that of other transition metals, such as $\operatorname{Ir}(631 \mathrm{~kJ} / \mathrm{mol}), \mathrm{Pt}(610$ $\mathrm{kJ} / \mathrm{mol})$, and $\mathrm{Rh}(580 \mathrm{~kJ} / \mathrm{mol})$ which form highly dispersed clusters on graphene layers [17]. As a result, the normalized XPS signal of ruthenium maintains an almost constant value, even though that of Pd gradually decreases. This observation is consistent with the IRAS results of adsorbed CO shown in Figure 2 (a). The agglomeration of Pd clusters reduces the number of adsorption sites of $\mathrm{CO}$ due to the reduction in surface area. Hence, the infrared intensity of $\mathrm{CO}$ gradually decreases without change in the pattern of IRAS spectrum. The IR intensity of adsorbed $\mathrm{CO}$ on the surface annealed at $900 \mathrm{~K}$ is reduced by $\sim 25 \%$ in comparison to that on the 
as-deposited surface. It is noteworthy that the vibrational feature of bridge-bound CO appears at $\sim 1975 \mathrm{~cm}^{-1}$ which is substantially higher than that of CO bridge-bound on $\operatorname{Pd}(111)$ terrace sites $\left(\sim 1950 \mathrm{~cm}^{-1}\right)$. This is characteristic of bridge-bound CO on defect-rich and/or highly-stepped $\operatorname{Pd}(111)$ facets [20]. In addition, the surfaces of alumina-supported palladium clusters (mean particle size $\sim 3.5 \mathrm{~nm}$ ) grown at $90 \mathrm{~K}$ have been shown to be very defective with no distinct facets, resulting in a blue-shifted vibrational feature of bridge-bound CO [20,21]. Hence, the CO vibrational feature appeared at $1975 \mathrm{~cm}^{-1}$ is a strong evidence for the formation of small palladium clusters on graphene at low Pd loadings. Moreover, it is interesting that the overall shape of the IR spectra did not change much, even though the annealing temperature of the sample was increased from 300 to $900 \mathrm{~K}$. The only difference observed was the decrease in the intensity of the $\mathrm{CO}$ vibrational feature. It indicates that the Pd cluster size on the graphene substrate slightly increases with increasing annealing temperature due to the weak interaction between palladium clusters and the graphene layer. Gotterbarm et. al [22] also observed the agglomeration of $\mathrm{Pd}$ on graphene/Rh(111) during annealing, where the interaction between graphene and substrate is weaker than that of graphene/Ru(0001). They proposed that $\mathrm{Pd}$ clusters are agglomerated on the graphene layer as the temperature was raised to $750 \mathrm{~K}$ since the XPS intensity of Pd gradually decreased and exhibited a minimum value at $750 \mathrm{~K}$ [17]. Further annealing caused the intercalation of Pd between the graphene layer and the Rh substrate [17]. In our study, the XPS intensity of $\mathrm{Pd}$ and the $\mathrm{Pd} / \mathrm{Ru}$ intensity ratio have both displayed minimum values at an annealing temperature of $900 \mathrm{~K}$, which is higher than that observed for the $\mathrm{Pd} / \mathrm{GR} / \mathrm{Rh}(111)$ system $(750 \mathrm{~K})$ [13]. This difference can be attributed to the stronger interaction between the graphene layer and the $\mathrm{Ru}(0001)$ substrate in our system, which results in higher energy needed for Pd to intercalate between the graphene layer and the Ru metal substrate. 
Above $900 \mathrm{~K}$ annealing temperature, however, the XPS intensity of Pd abruptly increases up to $1100 \mathrm{~K}$ and that of Ru concomitantly shows a gradual decrease. The IRAS spectrum of adsorbed CO shows a significant reduction in intensity after annealing at $1000 \mathrm{~K}$. Eventually, the CO vibrational feature completely disappears as the sample is annealed to $1100 \mathrm{~K}$. It is indicated that there is no Pd on graphene layer and the graphene layer does not have any defect sites at all. Even though the $\mathrm{CO}$ feature is completely disappeared in the IRAS, the XPS intensity of Pd restores almost to the same value as its original intensity was, as shown in Figure 2 (a). These observations are the direct evidences that the Pd deposited onto the graphene layer is intercalated between the graphene layer and the Ru substrate without any damage of graphene layer. The gradual decrease in XPS signal of Ru is demonstrated that spreading of the intercalated palladium layer occurred on the Ru substrate under the graphene layer. As mentioned above, the interaction between graphene and ruthenium is stronger than that of graphene and rhodium $[3,4,17]$, and therefore, the temperature at which intercalation of palladium occurs is higher in our system, than in the case of GR/Rh(111). Further annealing the sample above $1100 \mathrm{~K}$ causes the reduction of Pd and increase in Ru XPS intensities due to the onset of Pd desorption at this temperature, as it has been shown for Pd overlayers on refractory metals [23,24]. The onset of multilayer Pd desorption is $\sim 1100 \mathrm{~K}$, while Pd monolayer desorbs at 1300 K [23,24]. Therefore, the increase in the intensity of Ru XPS signal above $1100 \mathrm{~K}$ is attributed to the desorption of palladium. Despite desorption of a portion of the palladium intercalated between the graphene layer and the Ru substrate, the IRAS spectrum after CO exposure shows no vibrational features. These results unambiguously show that the graphene layer remained intact on the Ru substrate without any measurable damage during the processes of intercalation and desorption of Pd. Annealing at $1400 \mathrm{~K}$ causes the complete desorption of palladium from the $\mathrm{Ru}$ 
substrate and the dissolution of the graphene layer into Ru bulk. The Pd XPS feature completely disappears after annealing at $1400 \mathrm{~K}$ and the $\mathrm{CO}$ vibrational feature in the IRAS spectrum appears with high intensity at $2055 \mathrm{~cm}^{-1}$, assigned to adsorbed $\mathrm{CO}$ on $\mathrm{Ru}(0001)[25,26]$.

The thermal behavior of Pd clusters on graphene were further investigated as a function of Pd coverage. Panels (b) - (d) of Figure 1 show the normalized XPS intensities of Pd and Ru, as well as the $\mathrm{Pd} / \mathrm{Ru}$ intensity ratio for various coverages of $\mathrm{Pd}(1.0-5.0 \mathrm{MLE})$ on graphene as a function of annealing temperature, respectively. Despite different Pd coverages on graphene, the variation of XPS intensities of Pd shows a similar trend: it gradually decreases with increasing annealing temperature in the lower temperature region (300 - $900 \mathrm{~K})$. However, further annealing, but still below the desorption temperature of palladium, causes an increase in Pd XPS intensity. Eventually, at high annealing temperatures (above $1100 \mathrm{~K}$ ) a steep decrease in the XPS intensity of Pd is observed due to the onset of palladium desorption. At low Pd coverages $(\leq 1.0 \mathrm{MLE})$, the thermal behavior of the metal overlayers as a function of annealing temperature is very similar. However, as the Pd coverage increases above 1 MLE, the temperature having minimum Pd XPS intensity decreases and the variation of Pd intensity is more pronounced due to the facile agglomeration of large Pd clusters present on the graphene surface at high coverages of Pd. Panels (b) - (d) of Figure 2 show series of IRAS spectra of CO on several $\mathrm{Pd} / \mathrm{GR} / \mathrm{Ru}(0001)$ systems with different coverages of palladium under $\mathrm{CO}$ atmosphere $\left(1.0 \times 10^{-}\right.$ ${ }^{6}$ Torr) obtained at $300 \mathrm{~K}$ sample temperature. Over various coverage of Pd (1.0 - 5.0 MLE), IRAS spectra of adsorbed CO show very similar patterns as those of 0.25 MLE of $\mathrm{Pd} / \mathrm{GR} / \mathrm{Ru}(0001)$. At annealing temperatures up to $1000 \mathrm{~K}, \mathrm{CO}$ vibrational feature appears, but the intensity decreases with increasing annealing temperature. Between 1100 and $1300 \mathrm{~K}$, the $\mathrm{CO}$ vibrational feature completely disappears even under $\mathrm{CO}$ atmosphere. If the intercalation 
and subsequent desorption of palladium would cause any damage or loss to graphene layer we should be able to monitor it by the characteristic vibrational features of adsorbed $\mathrm{CO}$ on the exposed Ru metal sites. However, no evidence of any adsorbed $\mathrm{CO}$ is seen on the high temperature $(>1100 \mathrm{~K})$ annealed $\mathrm{Pd} / \mathrm{GR} / \mathrm{Ru}(0001)$ samples, not even for the highest Pd covered one (5.0 MLE). This observation clearly indicates the high structural stability of the graphene layer during the thermal stability study of Pd overlayers. The graphene layer remains intact without any physical damage during intercalation and subsequent desorption of the Pd overlayer. This result is corresponding to the exchange mechanism of intercalation. Jin et al. [9] reported that $\mathrm{Ni}$ adatoms anchoring on graphene layer may produce transient atomic-scale defects at the graphene lattice by the strong interaction between $\mathrm{Ni}$ and carbon and $\mathrm{Ni}$ diffused through this transient defects. After Ni adatoms pass though the graphene layer and intercalated, the transient defects are healed by itself and a perfect graphene surface is reformed [9]. Our IRAS results after intercalation of Pd under graphene and after the partial desorption of Pd do not show any $\mathrm{CO}$ vibrational features. If there is any extended defect or additional defect formed during the intercalation and/or desorption of $\mathrm{Pd}, \mathrm{CO}$ vibrational feature should be immediately appeared. This observation apparently shows that Pd pass through the graphene layer without any damage during the intercalation and the desorption from substrate. The differences in the IRAS spectra for samples with different Pd coverages arise primarily from the different initial sizes of the metal clusters formed as the Pd coverage was varied from 0.25 to 5.0 MLE. At the lowest Pd coverage of 0.25 MLE, almost exclusively one type of bridge-bound $\mathrm{CO}$ is present, which is represented by the IR feature centered at $1975 \mathrm{~cm}^{-1}$. Libuda et al. [27] previously reported that palladium clusters supported on alumina have $\operatorname{Pd}(111)$ and $\operatorname{Pd}(100)$ facets and they assigned the 1930 and $1840 \mathrm{~cm}^{-1}$ features to $\mathrm{CO}$ adsorbed on $\operatorname{Pd}(111)$, the $1960 \mathrm{~cm}^{-1}$ band to a superposition 
of bridge-bound $\mathrm{CO}$ on $\mathrm{Pd}(100)$ facets and $\mathrm{CO}$ adsorbed on defect sites, such as particle edges or steps, and the $2080 \mathrm{~cm}^{-1}$ feature is $\mathrm{CO}$ adsorbed in on-top geometry. As shown in Figure 2, every series of IR spectra shows one prominent feature at $1975-1990 \mathrm{~cm}^{-1}$ with low intensity features at $\sim 2085$ and $\sim 1930 \mathrm{~cm}^{-1}$. It clearly demonstrates the presence of small threedimensional palladium clusters on the graphene layer and this morphology maintained until palladium is intercalated. The decrease in IR intensity as a function of annealing temperature substantiates the agglomeration of Pd clusters as the annealing temperature increases. The Pd particles at low coverage (0.25 MLE) are small, and no dominant, large crystallographic planes ((100) and (111)) have developed. With increasing Pd coverage three trends are seen on the series of IR spectra: 1. The total integrated intensity of the IR features of adsorbed CO increases with Pd loading; 2. The position of the above discussed IR feature characteristic of bridge-bound CO on small Pd clusters gradually shifts to higher wavenumbers $\left(1975 \mathrm{~cm}^{-1}\right.$ at 0.25 MLE and $\sim 1990 \mathrm{~cm}^{-1}$ for the 5 MLE sample); and 3. Two new bands develope: one at a lower frequency (characteristic of another type of bridge-bond CO), and one at higher frequency (representing linearly adsorbed $\mathrm{CO}$ ). The overall intensity increase of the IR features of adsorbed $\mathrm{CO}$ is in concert with the increase in available adsorption sites as the amount of Pd deposited increases. The changes in the IR peak positions and the development of two new absorption features as the amount of Pd deposited increases are direct consequences in the change of the Pd clusters (both sizes and shapes). As the amount of Pd deposited increases large Pd clusters with different crystallographic planes form, and the adsorbed $\mathrm{CO}$ probes these different sites.

\section{Conclusions}


The thermal behaviors of $\mathrm{Pd}$ overlayers at various coverages on graphene/Ru(0001) have been investigated as a function of annealing temperature using surface spectroscopic techniques. For all Pd coverages studied three distinct processes were identified: annealing the room temperature-deposited metal overlayers to $\sim 900 \mathrm{~K}$ resulted in the agglomeration of Pd clusters. In the temperature range of $\sim 900-1100 \mathrm{~K} \mathrm{Pd}$ intercalated between the graphene layer and the $\mathrm{Ru}(0001)$ substrate. Above $1100 \mathrm{~K}$ Pd begins to desorb, and by $1400 \mathrm{~K}$ all the Pd overlayer was removed by desorption, while the graphene layer dissolved into the metal substrate. These processes were clearly evidenced from both the variation in the intensities of the metal XPS signals and the IRAS spectra of adsorbed CO during annealing in vacuum. The IRAS feature of bridge-bound $\mathrm{CO}$ on Pd/graphene blue-shifted with increasing Pd coverage demonstrating that small and highly stepped clusters are formed during the deposition of Pd at room temperature. Due to the weak interaction between Pd and graphene agglomeration of Pd clusters occurred at low annealing temperatures. However, higher temperature annealing (but below the desorption temperature of $\mathrm{Pd}$ ) results in the intercalation of palladium between graphene and ruthenium, and at even higher temperature the intercalated Pd desorbs and the graphene layer is dissolved in $\mathrm{Ru}$ substrate. Interestingly, no noticeable structural changes were observed in the graphene layer either during intercalation or Pd desorption.

\section{Acknowledgments}

We gratefully acknowledge the US Department of Energy (DOE), Office of Science, Office of Basic Energy Sciences, Chemical Sciences, Geosciences, and Biosciences Division for the support of this work. The research described in this article was performed at the Environmental Molecular Sciences Laboratory (EMSL), a national scientific user facility 
sponsored by the DOE Office of Biological and Environmental Research and located at Pacific Northwest National Laboratory (PNNL). PNNL is operated for the US DOE by Battelle Memorial Institute under contract number DE-AC05-76RL01830. This work was also supported by the Sungshin Women's University Research Grant of 2014. 


\section{References}

[1] M. Batzill, Surf. Sci. Rep. 67 (2012) 83-115.

[2] A. Dahal, M. Batzill, Nanoscale 6 (2014) 2548-2562.

[3] K. Gotterbarm, C. Bronnbauer, U. Bauer, C. Papp, H.P. Steinruck, J. Phys. Chem. C 118 (2014) 25097-25103.

[4] K. Gotterbarm, C. Steiner, C. Bronnbauer, U. Bauer, H.P. Steinruck, S. Maier, C. Papp, J. Phys. Chem. C 118 (2014) 15934-15939.

[5] Q. Liao, H.J. Zhang, K. Wu, H.Y. Li, S.N. Bao, P. He, Surf. Rev. Lett. 19 (2012) 5.

[6] M. Liu, R. Zhang, W. Chen, Chemical Reviews 114 (2014) 5117-5160.

[7] C. Tan, X. Huang, H. Zhang, Materials Today 16 (2013) 29-36.

[8] Y. Cui, J.F. Gao, L. Jin, J.J. Zhao, D.L. Tan, Q. Fu, X.H. Bao, Nano Res. 5 (2012) 352-360.

[9] L. Jin, Q. Fu, Y. Yang, X.H. Bao, Surf. Sci. 617 (2013) 81-86.

[10] C.-W. Yi, J.H. Kwak, C.H.F. Peden, C. Wang, J. Szanyi, J. Phys. Chem. C 111 (2007) 14942-14944.

[11] C.-W. Yi, J.H. Kwak, J. Szanyi, J. Phys. Chem. C 111 (2007) 15299-15305.

[12] C.-W. Yi, J. Szanyi, J. Phys. Chem. C 113 (2009) 716-723.

[13] C.-W. Yi, J. Szanyi, J. Phys. Chem. C 113 (2009) 2134-2140.

[14] S. Axnanda, K.D. Cummins, T. He, D.W. Goodman, M.P. Soriaga, ChemPhysChem 11 (2010) 14681475.

[15] C.W. Yi, K. Luo, T. Wei, D.W. Goodman, J. Phys. Chem. B 109 (2005) 18535-18540.

[16] S.R. Wang, Y. Zhang, N. Abidi, L. Cabrales, Langmuir 25 (2009) 11078-11081.

[17] Z.H. Zhou, F. Gao, D.W. Goodman, Surf. Sci. 604 (2010) 1071-L1038.

[18] A.T. N'Diaye, S. Bleikamp, P.J. Feibelman, T. Michely, Physical Review Letters 97 (2006).

[19] A.T. N'Diaye, T. Gerber, C. Busse, J. Myslivecek, J. Coraux, T. Michely, New Journal of Physics 11 (2009).

[20] H. Unterhalt, G. Rupprechter, H.-J. Freund, J. Phys. Chem. B 106 (2001) 356-367.

[21] I.V. Yudanov, et al., J. Phys. Chem. B 107 (2002) 255-264.

[22] K. Gotterbarm, W. Zhao, O. Hofert, C. Gleichweit, C. Papp, H.P. Steinruck, Phys. Chem. Chem. Phys. 15 (2013) 19625-19631.

[23] D.E. Pierce, R.P. Burns, K.A. Gabriel, Thin Solid Films 206 (1991) 340-344.

[24] J. Storm, R.M. Lambert, N. Memmel, J. Onsgaard, E. Taglauer, Surf. Sci. 436 (1999) 259-268.

[25] H. Hartmann, T. Diemant, J. Bansmann, R.J. Behm, Surf. Sci. 603 (2009) 1456-1466.

[26] H. Pfnür, D. Menzel, F.M. Hoffmann, A. Ortega, A.M. Bradshaw, Surf. Sci. 93 (1980) 431-452.

[27] J. Libuda, H.J. Freund, Surf. Sci. Rep. 57 (2005) 157-298. 


\section{Figure Captions}

Figure 1. Normalized XPS intensities of $\mathrm{Pd}, \mathrm{Ru}$, and the ratio of $\mathrm{Pd} / \mathrm{Ru}$ for (a) $0.25 \mathrm{MLE}$, (b) 1.0 MLE, (c) 2.0 MLE, and (d) 5.0 MLE Pd/graphene/Ru(0001) as a function of annealing temperature. The palladium was deposited at room temperature.

Figure 2. IRAS spectra of adsorbed CO on (a) 0.25 MLE, (b) 1.0 MLE, (c) 2.0 MLE, and (d) 5.0 $\mathrm{MLE} \mathrm{Pd/graphene/Ru(0001)} \mathrm{systems} \mathrm{at} \mathrm{a} \mathrm{CO} \mathrm{pressure}\left(1.0 \times 10^{-6} \mathrm{Torr}\right)$ as a function of annealing temperature. All the IR spectra were taken at room temperature.

Figure S1. Calibration curve for Pd deposition: $\mathrm{Pd} / \mathrm{Ru}$ XPS signal intensity ratio as a function of deposition time at a constant rate of metal evaporation.

Figure S2. LEED pattern for pristine graphene at $70.4 \mathrm{eV}$ (a) and $15.4 \mathrm{eV}$ (b).

Figure S3. Series of XP spectra of Pd 3d (panel a) and Ru 3d (panel b) collected during the stepwise annealing of a $1 \mathrm{MLE} \mathrm{Pd} / \mathrm{GR} / \mathrm{Ru}(0001)$ sample. 


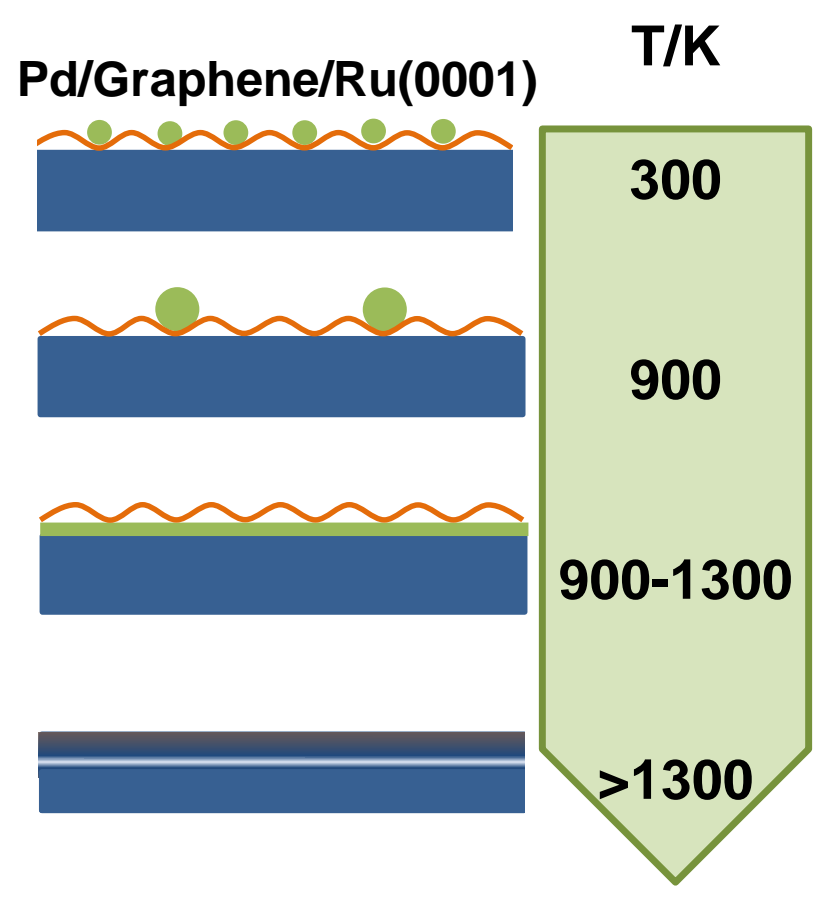

